\title{
Multifunctional Nanomaterials for Biomedical Engineering: Unique Properties, Fabrications, and Diverse Applications
}

\author{
Jen-Jie Chieh, ${ }^{1}$ Sharmila M. Mukhopadhyay, ${ }^{2}$ and Yali Cui ${ }^{3}$ \\ ${ }^{1}$ Institute of Electro-Optical Science and Technology, National Taiwan Normal University, Taipei 116, Taiwan \\ ${ }^{2}$ Mechanical \& Materials Engineering, Wright State University, Dayton, OH 45324, USA \\ ${ }^{3}$ National Engineering Research Center for Miniaturized Detection Systems, Xian, Shaanxi 710069, China
}

Correspondence should be addressed to Jen-Jie Chieh; jjchieh@ntnu.edu.tw

Received 3 May 2015; Accepted 3 May 2015

Copyright (c) 2015 Jen-Jie Chieh et al. This is an open access article distributed under the Creative Commons Attribution License, which permits unrestricted use, distribution, and reproduction in any medium, provided the original work is properly cited.

Nanomaterials possess many unique properties useful for biomedical applications ranging from preoperative and intraoperative imaging to coatings, assays, and cell scaffolds. Their popularity and production value are full of competitiveness. Their related methodologies and instruments are also created or emphasized as the mainstream. Since multifunctional nanomaterials attract interests and attention of researchers, engineers, and businessmen in biomedical engineering, this journal is set up to publish a special issue devoted to this topic. The result is a collection of five original research articles, whose authors belong to academic or research institutions of five different countries from Asia, Europe, and America. Papers are representative of a large share of biomaterials with multiple functions, applications and syntheses, and characteristic methods.

From a scientific and engineering point of view, the perspective is to promote merits of biomaterials. S.-H. Liao et al. from Taiwan ("Assaying Biomarkers via RealTime Measurements of the Effective Relaxation Time of Biofunctionalized Magnetic Nanoparticles Associated with Biotargets") proposed two different methods for assaying alpha-fetoprotein biomarkers of liver cancers based on the same biofunctionalized magnetic nanoparticles. One is the effective relaxation time by the ac magnetic susceptometer, and the other is saturation magnetization by the vibrating sample magnetometer. Both showed the high future clinical possibility. E. M. F. Lemos et al. from Brazil ("Comparison of the Effect of Sol-Gel and Coprecipitation Routes on the Properties and Behavior of Nanocomposite Chitosan-Bioactive Glass Membranes for Bone Tissue Engineering") developed hybrid composite films for achieving adequate performance for the application as a guide to the cell growth of bone tissue. There were two synthetic routes of sol-gel and coprecipitation by combining chitosan (CS) with bioactive glass in solution form (BG-Sol) and in nanoparticle dispersion form (BGNP) by the two different synthesis routes. Both systems of CSBG as CS-BGNP films exhibited high tensile strength, high bioactivity, and cell viability. These results indicate that the use of bioactive glass successfully acted as an agent for loading and improved bioactivity, particularly in the form of nanoparticle dispersion (BGNP), whose results are the most promising. R. Pinna et al. from Italy ("Effectiveness of Two Self-Etchings Bonded Clinically in Caries Affected Dentin with Homogeneous Smear Layer") characterized the interface of two self-etching adhesives bonded in carious affected dentin with homogeneous smear layer. The observation of nanoscale structures by TEM was used to study different factors, such as different two-step self-etching systems and clinical conditions of pulpal pressure. Hence, this work originally proposed the correlate self-etchings with the heterogeneous aspects of vital carious affected dentin, which occur in daily practice under clinical conditions. $\mathrm{K}$. W. Huang et al. from Taiwan ("Time-Evolution Contrast of Target MRI Using High-Stability Antibody Functionalized Magnetic Nanoparticles: An Animal Model") developed the surface biofunctions, $\mathrm{Fe}_{3} \mathrm{O}_{4}$ magnetic nanoparticles for the ability of immunoassay by immunomagnetic reduction, and in vivo target labeling by magnetic resonance imaging. This work verified the application of nanoparticles to both MRI contrast and immunoassay of a colorectal cancer. 
C. L. Popa et al. from Romania ("Physicochemical Analysis of the Polydimethylsiloxane Interlayer Influence on a Hydroxyapatite Doped with Silver Coating") described the captivating physico-chemical process during the interaction of a silver doped hydroxyapatite coating with a polydimethylsiloxane layer was investigated by different complementary methods, such as the X-ray diffraction, Fourier Transform Infrared Spectroscopy, Scanning Electron Microscopy, and Glow Discharge Optical Emission Spectrometry. It indicated that some phenomenon could be characterized or found consistently by different methodologies. For example, the FT-IR analysis, in agreement with the XRD measurements, showed that the physical procedure used for the generation of the Ag:HAp-PDMS composite layer is useful for the formation of $\mathrm{SiO}_{4}{ }^{4-}$ ions. The X-ray diffraction and Fourier Transform Infrared Spectroscopy measurements show that the hydroxyapatite doped with silver is in a crystalline form and some $\mathrm{SiO}_{4}{ }^{4-}$ ions formation takes place at the surface and in the bulk of the new hydroxyapatite doped with silver/polydimethylsiloxane composite layer. Uniformly, the authors highlighted the potentiality of this emerging field of biomaterials related to its multiple functions, applications and syntheses, and characteristic methods.

Jen-Jie Chieh

Sharmila M. Mukhopadhyay Yali Cui 

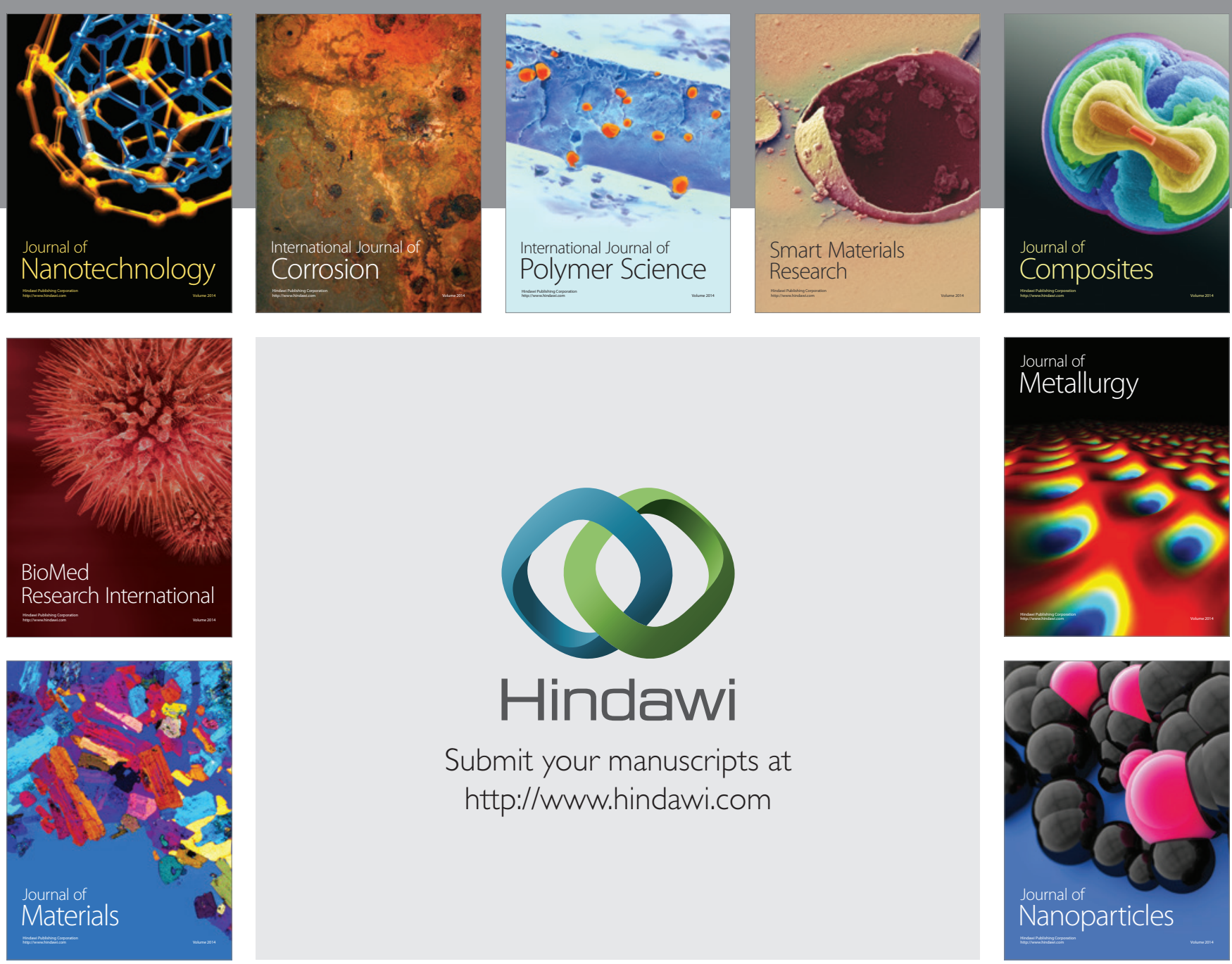

Submit your manuscripts at http://www.hindawi.com
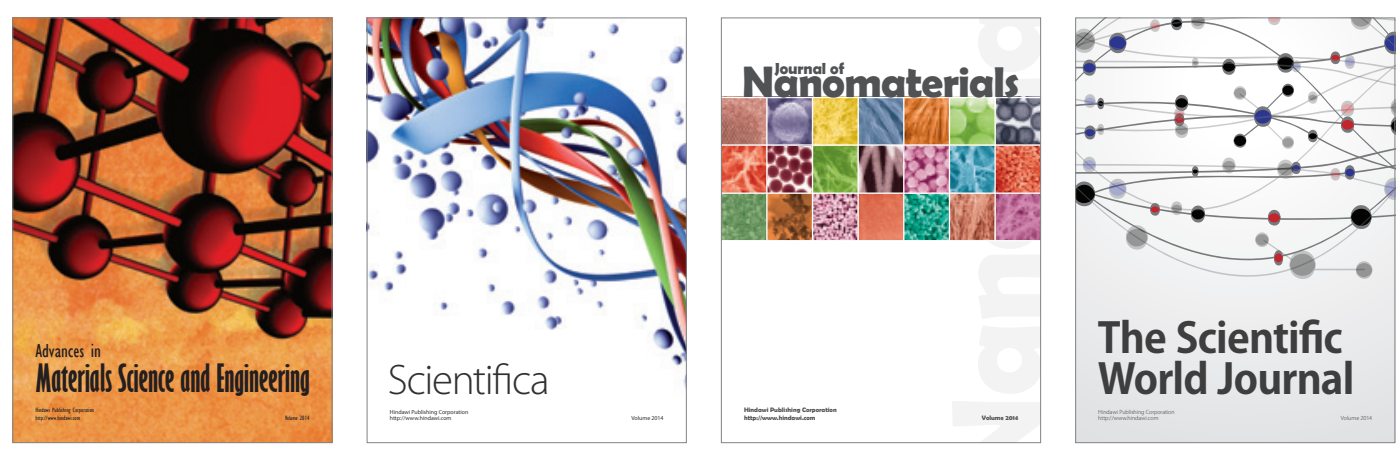

\section{The Scientific World Journal}
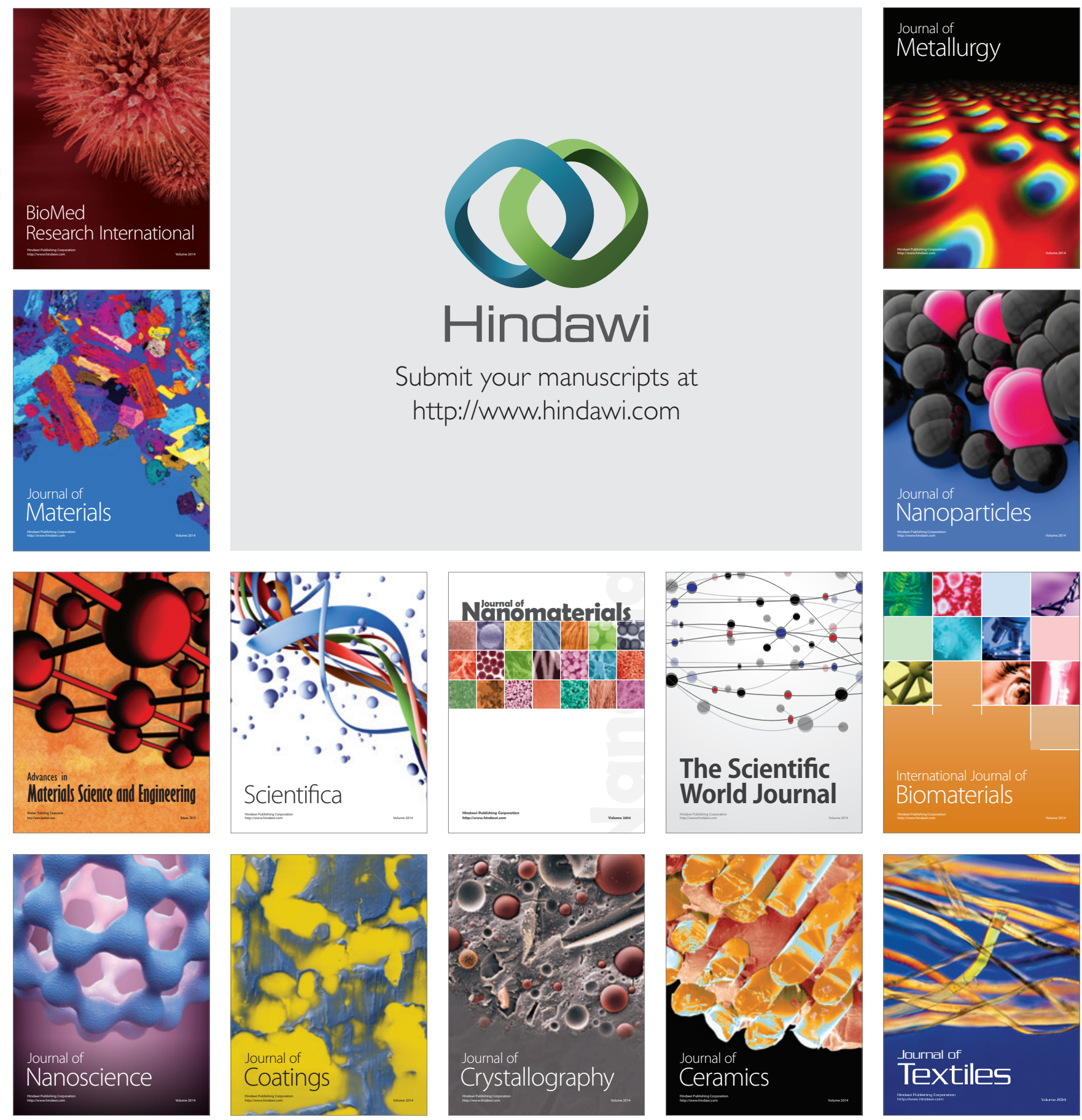